\title{
Psychological distress and health-related quality of life in implantable cardioverter defibrillator patients with coronary artery disease
}

\author{
Wong MF Florence \\ School of Nursing \\ Tung Wah College \\ Hong Kong \\ florencewong@twc.edu.hk
}

\begin{abstract}
Implantable cardioverter defibrillator (ICD) patients are found to have poorer health-related quality of life (HRQoL) and heightened psychological distress, particularly depression. Coronary artery disease (CAD) is evidenced to be associated with lowered HRQoL and more negative emotions. This study was to examine psychological status and HRQoL as well as identify factors associated with these two areas among ICD patients with a history of CAD. Of 57 ICD patients with CAD, most of them $(91.2 \%)$ aged 60 or older with mean age 63.04 years old $(\mathrm{SD}=14.58)$. A majority of them were married $(84.2 \%)$ and selfcare dependent $(89.5 \%)$. All participants had a history of SCA experience. Almost $80 \%$ were the first ICD recipients and had ICD implantation more than one year. Only two of them had ICD shock experience. The participants were more depressive (mean 3.67 \pm 3.57 ). About $8.8 \%$ of patients had severe clinical depression (score $\geq 8$ ) and $19.3 \%$ had severe clinical anxiety (score $\geq 8$ ). The participants had relatively poorer mental health (mean 58.35 \pm 9.31 ) than physical health (mean 38.15 \pm 14.98 ). Female gender was found to have positive association with anxiety; whereas, having a history of $\mathrm{DM}$ has positive association with depression. Self-care dependence was the most significant factor associated with anxiety and depression. Depression was found to be negatively associated with physical health; whereas, anxiety was negatively associated with mental health. By contrast, having occupation and having a history of hyperlipidemia were found to be positively associated with physical health. The results showed that identification of factors enables nurses to develop strategies to remove or reduce the negative effects but to provide more support to foster the positive effects arising from the factors. The study results can direct healthcare policy makers implement strategies for optimizing HRQoL and reducing psychological burdens.
\end{abstract}

Keywords-implantable cardioverter defibrillator; coronary artery disease; psychological distress; depression; anxiety; health-related quality of life

\section{INTRODUCTION}

Implantable cardioverter defibrillator (ICD) is an internal device to detect life-threatening arrhythmias, commonly ventricular tachycardia (VT) and ventricular fibrillation (VF), and administer immediate treatment in order to restore normal rhythms (Koplan 2009, Kwok et al. 2003). Patients at high risk of sudden cardiac arrest (SCA) are more likely to receive ICD implantation to increase survival rate. Coronary artery disease (CAD) has been accountable for the major portion of the mortality resulting from SCA (World Health Organization [WHO], 2016) and in Hong Kong (Centre for Health Protection [CHP], 2016). In 2012, there were more than 17 million people died from cardiovascular diseases that were about $30 \%$ of all global death (WHO, 2016). Of these deaths, there were more than 7 million due to CAD (WHO, 2016). In the US, CAD has been accountable for about 360 thousand of deaths each year (American Heart Association [AHA], 2017). In Hong Kong, CAD made up almost $67 \%$ of heart diseases deaths in 2014 (CHP, 2016). It is the most concern that CAD results in increased healthcare expenditure and physical disability (de Jong et al. 2006). The advanced medical technology and treatment together with improved cardiac rehabilitative scheme promotes recovery, shortens hospitalization, reduces rehospitalization frequency, prolongs survival rates, prevents severe conditions, and ensures optimal health-related quality of life (HRQoL). Therefore, there has been a huge rise in the total healthcare expenditure to CAD patients.

However, much evidence showed that patients with CAD patients experienced more psychological distress that is more likely to further lower their HRQoL (Schron et al. 2002, Carroll \& Hamilton 2008, Wong et al. 2014). Anxiety and depression are widely found in cardiac patients (Glassman 2007, Lichtman et al. 2008). Depression was accountable for estimated $10 \%$ to $20 \%$ of post-myocardial infarction (MI) patients (Tsu 2012). Estimated 31 to $45 \%$ of patients with CAD suffered from clinically significant depression (Thombs et al. 2006). Patients with depression after MI attack had greater risk of mortality, adjustment problems, and poorer HRQoL compared with those who without depression (de Jonge et al. 2006). Depression is evidenced as a major cardiac marker of CAD (Glassman 2007) and is accountable to two-tofourfold increase in mortality (Welin et al. 2000). It is important to note that CAD patients are also closely associated with the occurrence of life threatening incidence, commonly VT and VF (Koplan 2009, Kwok et al. 2003). Therefore, psychological distress, anxiety and particularly depression, is a great concern in CAD patients with a higher morbidity and mortality (Welin et al. 2000, Egede 2007, Freedenberg et al. 2011).

HRQoL embraces multidimensional aspects of well-being including physical, mental, and social domains, that reflects individual health as a whole considering various correlates,

DOI: $10.5176 / 2345-7198 \_5.1 .30$

ISSN 2345-7198

CThe Author(s) 2018. This article is published with open access by the GSTF 
such as health risks and socioeconomic status (Centers for Disease Control and Prevention [CDC] 2017). HRQoL is useful to evaluate the effects of medical treatment and healthcare outcomes (Issa et al. 2010). The past studies reported that CAD patients experienced poorer HRQoL (Tofighi et al. 2012, Unsar et al. 2007). Moreover, CAD patients with depression had a remarkable rise in impaired HRQoL (Moryś et al. 2016, Watkins et al. 2013, Yu et al. 2009). Since ICD has lowered HRQoL (Carroll \& Hamilton 2008, Goldenberg \& Moss 2008) and heightened psychological distress (Friedmann et al. 2006, Sears \& Conti 2002, Smolderen 2017). A variety of factors associated with HRQoL in ICD patients were reported and depression was found to be the most significant factor lowering HRQoL among ICD patients (Wong et al 2014). Since CAD patients are at high risk of sudden cardiac arrest, ICD patients may also have a history of CAD. Although psychological distress and HRQoL had been investigated previously, it is uncertain how psychological status of ICD patients with a history of CAD and how they perceive HRQoL outcomes. Therefore, this study aimed to examine psychological status and HRQoL outcomes in this specific population; and thus factors associated with psychological distress, including anxiety and depression, and HRQoL impacts would be identified to accurately direct strategies to improve ICD care in current clinical settings.

\section{METHODS}

\section{A. Design and sampling}

This study was adopted a cross-sectional design with a convenience sample. All eligible participants, who were adult ICD patients with a history of CAD, Hong Kong residents, and able to communicate with Chinese, were invited to join this study. A total of 57 ICD patients with a history of CAD were recruited at two regional hospitals.

\section{B. Data collection}

The eligible participants were required to fill in a set of questionnaire, including a demographic and clinical form, the Hong Kong Chinese-Cantonese version of Hospital Anxiety and Depression Scale (HADS), the Short Form-36 Health Survey version 2 (SF-36v2), was used for a structured face-toface interview. The interview took about 20 minutes.

The HADS was developed by Leung et al. (1999). It was used to measure anxiety (HAD-A) and depression (HAD-D). Each subscale consists of seven items rated from 0 to 3. A cut off score $\geq 8$ indicates clinical significant levels of anxiety and depression. The Cronbach's alphas of HAD-A, HAD-D, and HADS were $0.79,0.72$, and 0.83 respectively, indicating good reliability.

The SF-36v2 developed by Ware, et al. (1993) was used to measure HRQoL. It includes eight domains. These subscales scores can be aggregated to two summary scores: a physical component summary (PCS) and a mental component summary (MCS). The domain with higher scores indicates better HRQoL and vice versa. The Cronbach's alphas of SF-36v2 was 0.85 indicating very good reliability.

\section{ETHICAL CONSIDERATIONS}

The study was approved by the Research Ethics Committees of two study hospitals. All participants who agreed to join this study were requested to sign an informed consent. They were assured that they could withdraw from the study without consequences at any time. All information was anonymous to protect personal confidentiality and only used for research purpose.

\section{DATA ANALYSIS}

Data were analyzed using the IBM SPSS v20.0 (SPSS Inc., Chicago, IL, USA). Descriptive statistics were used to summarize and analyze the independent variables related to participants' demographic and clinical characteristics, HRQoL subscales, anxiety and depression. Pearson's coefficient, independent-samples t-test, or one-way ANOVA was used to examine the relationships between variables. Those independent variables with $\mathrm{p}$ values $<0.25$ were analyzed using stepwise regression to identify independent predictors associated with anxiety, depression, or HRQoL (PCS and MCS). The level of significance was set at 0.05 for all statistical tests.

\section{RESULTS}

\section{Demographic charcteristics}

Of a total of 57 ICD patients with a history of CAD, all of them were SCA survivors. Most of them (91.2\%) aged 60 or older with mean age 63.04 years old $(\mathrm{SD}=14.58)$. More than $80 \%$ of them were male and married. About $90 \%(n=52)$ were living with their family and self-care independent. Almost $88 \%$ of them were retired or unemployed. The demographic characteristics are showed in Table 1.

TABLE I. Demographics

\begin{tabular}{|l|c|c|}
\hline Variables & Number & Percentage \\
\hline Age & & \\
\hline$<50$ & 3 & 5.3 \\
\hline $50-59$ & 2 & 3.5 \\
\hline $60-69$ & 26 & 45.6 \\
\hline$\geq 70$ & 26 & 45.6 \\
\hline Gender & & \\
\hline Male & 46 & 80.7 \\
\hline Female & 11 & 19.3 \\
\hline Marital status & & \\
\hline Single & 9 & 15.8 \\
\hline Married & 48 & 84.2 \\
\hline Living status & & \\
\hline Alone & 5 & 8.8 \\
\hline With family & 52 & 91.2 \\
\hline Self-care dependence & & \\
\hline No & 51 & 89.5 \\
\hline Yes & 6 & 10.5 \\
\hline Educational level & & \\
\hline Uneducated & 5 & 8.8 \\
\hline Primary school & 20 & 35.1 \\
\hline Secondary school & 23 & 40.4 \\
\hline Tertiary or above & 9 & 15.8 \\
\hline Occupation & & \\
\hline Retired/ unemployed & 50 & 87.7 \\
\hline Employed & 7 & 12.3 \\
\hline
\end{tabular}




\section{Clinical charcteristics}

Of a total of 57 ICD patients with CAD, more than half of them had heart failure (HF), hypertension (HT), and hyperlipidemia but only $28 \%$ had atrial fibrillation (AF). About $39 \%$ of them had diabetes mellitus (DM). The clinical characteristics are showed in Table II.

TABLE II. Clinical characteristics

\begin{tabular}{|c|c|c|}
\hline Variables & Number & Percentage \\
\hline Heart failure (HF) & & \\
\hline No & 24 & 42.1 \\
\hline Yes & 33 & 57.9 \\
\hline Hypertension (HT) & & \\
\hline No & 25 & 43.9 \\
\hline Yes & 32 & 56.1 \\
\hline Atrial fibrillation (AF) & & \\
\hline No & 41 & 71.9 \\
\hline Yes & 16 & 28.1 \\
\hline Hyperlipidemia & & \\
\hline No & 26 & 45.6 \\
\hline Yes & 31 & 54.4 \\
\hline Diabetes mellitus (DM) & & \\
\hline No & 35 & 61.4 \\
\hline Yes & 22 & 38.6 \\
\hline
\end{tabular}

\section{E. ICD-related characteistics}

Of 57 ICD patients with CAD, more than half of them had received conventional ICD. Most of them (77.2\%) were first ICD recipients and had undergone ICD implantation for more than one year. However, all but only two had not had ICD shock experience. The ICD-related characteristics are showed in Table III.

TABLE III. ICD-related characteristics

\begin{tabular}{|l|c|c|}
\hline Variables & Number & Percentage \\
\hline Types of ICD & & \\
\hline Conventional ICD & 32 & 56.1 \\
\hline CRT-D & 25 & 43.9 \\
\hline Duration of ICD implantation & & \\
\hline$<12$ months & 13 & 22.8 \\
\hline$\geq 12$ months & 44 & 77.2 \\
\hline Number of ICD implantation & & \\
\hline$<1$ & 45 & 78.9 \\
\hline$\geq 2$ & 12 & 21.1 \\
\hline ICD shock experience & & \\
\hline No & 55 & 96.5 \\
\hline Yes & 2 & 3.5 \\
\hline
\end{tabular}

F. Psychological status and HRQoL

Based on the HADS, the means of HAD-A, HAD-D, and HADS were $2.75(\mathrm{SD}=3.52), 3.67(\mathrm{SD}=3.57)$, and 6.42 $(\mathrm{SD}=6.26)$ respectively. Of $57 \mathrm{ICD}$ patients with $\mathrm{CAD}$, about 9\% and $19.4 \%$ of patients had clinically significant anxiety and depression respectively. About $9 \%$ of patients had severe clinical anxiety (cut-off point at score $\geq 8$ ) and about $19 \%$ had severe clinical depression (cut-off point at score $\geq 8$ ). Moreover, the results of SF-36v2 showed that the means of PCS and MCS were $38.15(\mathrm{SD}=14.98)$ and $58.35(\mathrm{SD}=9.31)$ respectively. The psychological status and HRQoL are showed in Table IV.

TABLE IV. Psychological status and HRQoL

\begin{tabular}{|l|l|l|}
\hline & Number & Percentage \\
\hline
\end{tabular}

\begin{tabular}{|c|c|c|}
\hline Anxiety (score $\geq \mathbf{8}$ ) & 5 & 8.8 \\
\hline Depression (score $\geq \mathbf{8}$ ) & 11 & 19.3 \\
\hline HADS & Mean & SD \\
\hline HAD-A & 6.42 & 6.26 \\
\hline HAD-D & 2.75 & 3.52 \\
\hline HRQoL & 3.67 & 3.57 \\
\hline PCS & & \\
\hline MCS & 38.15 & 14.98 \\
\hline
\end{tabular}

G. Predictors of anxiety and depression

The results showed that anxiety was significantly associated with female gender $(B=3.518, S E=1.050, p=0.001)$ and self-care dependence $(\mathrm{B}=3.358, \mathrm{SE}=1.350, \mathrm{p}=0.016)$ $[\mathrm{F}(2,54)=8.527, \quad \mathrm{p}<0.001]$. Moreover, depression was significantly associated with self-care dependence $(B=3.724$, $\mathrm{SE}=1.336, \mathrm{p}=0.007)$ and $\mathrm{DM}(\mathrm{B}=2.139, \mathrm{SE}=0.836, \mathrm{p}=0.013)$ $[\mathrm{F}(5,51)=4.640, \mathrm{p}=0.001]$. The analytic results of multivariate regression are showed in Table $\mathrm{V}$.

TABLE V. Predictors of anxiety and depression

\begin{tabular}{|c|c|c|c|c|c|c|}
\hline & \multicolumn{3}{|c|}{ Anxiety } & \multicolumn{3}{c|}{ Depression } \\
\cline { 2 - 7 } & B & SE & p & B & SE & p \\
\hline Gender & & & & & & \\
\hline Male (ref) & & & & & & \\
\hline Female & 3.518 & 1.050 & 0.001 & & & \\
\hline $\begin{array}{l}\text { Self-care } \\
\text { dependence }\end{array}$ & & & & & & \\
\hline No (ref) & & & & & & \\
\hline Yes & 3.358 & 1.350 & 0.016 & 3.724 & 1.336 & 0.007 \\
\hline DM & & & & & & \\
\hline No (ref) & & & & & & \\
\hline Yes & & & & 2.139 & 0.836 & 0.013 \\
\hline
\end{tabular}

\section{H. Predictors of PCS and MCS}

The results showed that PCS was negatively associated with depression $(B=-2.384, \mathrm{SE}=0.423, \mathrm{p}<0.001)$ but positively associated with occupation $(B=11.815, S E=4.640, p=0.014)$ and hyperlipidemia $(\mathrm{B}=7.295, \quad \mathrm{SE}=2.970, \quad \mathrm{p}=0.017)$ $[\mathrm{F}(3,53)=17.299, \mathrm{p}<0.001]$. Moreover, MCS was found negatively associated with anxiety $(\mathrm{B}=-1.628, \mathrm{SE}=0.281$, $\mathrm{p}<0.001)[\mathrm{F}(1,55)=33.625, \mathrm{p}<0.001]$. The analytic results of multivariate regression are showed in Table VI.

TABLE VI. Predictors of PCS and MCS

\begin{tabular}{|c|c|c|c|c|c|c|}
\hline & \multicolumn{3}{|c|}{ PCS } & \multicolumn{3}{c|}{ MCS } \\
\cline { 2 - 7 } & B & SE & p & B & SE & p \\
\hline Anxiety & & & & -1.628 & 0.281 & $<0.001$ \\
\hline Depression & -2.384 & 0.423 & $<0.001$ & & & \\
\hline Occupation & & & & & & \\
\hline No (ref) & & & & & & \\
\hline Yes & 11.815 & 4.640 & 0.014 & & & \\
\hline Hyperlipidemia & & & & & & \\
\hline No (ref) & & & & & & \\
\hline Yes & 7.295 & 2.970 & 0.017 & & & \\
\hline
\end{tabular}

\section{DISCUSSION}

Our study aimed at examining the psychological status and HRQoL and identifying factors associated with these two areas in ICD patients with a history of CAD. In this study, the number of the participants had severe clinical depression $(\sim 19 \%)$ and severe clinical anxiety $(8.8 \%)$. The participants reported higher level of depression that can be due to their previous experience of SCA or unpredictable cardiac attack. 
Moreover, most of the participants were the first ICD recipients indicating that they are still adapting to live with their ICD device (Wong et al. 2014). The results of depression and anxiety levels of the participants in this study were relatively lower than other ICD patients (16.2-50.6\% and 37.5-59.4\% respectively) (Bilge et al. 2006, Friedmann et al. 2006, Thomas et al. 2006). It is important to note that psychological distress, anxiety and particularly depression, are presented differently in cardiac patients when their history of CAD is also considered. It indicates that the needs of patients are various among different types of cardiac patients.

In this study, self-care dependence was found to be the most significant factor associated with both anxiety and depression. Female gender was found to be positively associated with anxiety; whereas, having a history of DM would perceive more depression. Self-care dependence refers to an individual ability to perform daily activities of living (Easton 1993, Sidani 2003). Loss of self-care independence increases anxiety and depression most probably due to low self-esteem and helplessness (Wong et al. 2014). ICD patients with CAD may feel different from others as they need to rely on ICD device for life (Josephson \& Wellens 2004). Some physical restrictions from ICD and poor cardiac condition may reduce self-care ability leading to more psychological distress in the ICD patients with CAD. Female ICD patients relatively feel more anxious most probably due to low self-image and low self-esteem resulting from bodily changes after ICD implantation (Vazquez et al 2008). Most of the female participants in the present study were housewives or retired. Their ICD and CAD experience can increase physical restriction leading to heightened psychological distress, particularly anxiety. ICD patients with CAD who have a history of DM perceived more depressed probably due to poor control of blood glucose level and deteriorated general health condition (Porojan et al. 2012).

Furthermore, better mental health was revealed among the participants in the present study compared to other CAD patients. The participants in this study perceived relative better mental health (mean 58.35 versus mean 49.89 respectively) but poorer physical health (mean 38.15 versus mean 49.72 respectively) than other CAD patients (Tofighi et al. 2012). It can be explained that CAD patients perceived poorer mental health as they are uncertain about the deteriorating changes in health condition. They may be worried about the episodes of sudden cardiac attack and dying. In ICD patients with CAD, they will feel safe to live with the ICD as they perceive that ICD is lifesaver (Josephson \& Wellens 2004). On the contrary, the physical health is better in other CAD patients most probably because they were treated and educated well to improve their cardiac wellness, such as medication adherence, lifestyle modification and cardiac rehabilitation. However, ICD patients with CAD will perceive poorer physical health most probably due to the physical restriction in order to maintain the normal ICD operation and prevent inappropriate shocks (Carroll \& Hamilton 2005, Schron et al. 2012).

ICD patients with a history of CAD who had higher degree of depression will perceive poorer physical health. Depression was reported to be the most significant factor associated with mental and physical health (Wong et al. 2014). Previous studies also supported that depression may increase physical disability (Carroll \& Hamilton, 2005, Hallas et al. 2010). It can be due to the fact the depression induces physiological responses resulting from sympathetic nervous stimulation leading to increased cardiac workload. Moreover, having an occupation and having a history of hyperlipidemia were found to be associated with better physical health. In the present study, the participants who are more anxious will perceive poorer mental health. As discussed previously, ICD patients with a history of CAD may perceive more psychological distress due to their poor health condition and perception of living with their ICD for life. The participants who had higher degree of anxiety perceived poorer mental health. It can be explained the fact that ICD patients with a history of CAD can feel their ICD device as a reminder of their previous experience and reoccurrence of sudden cardiac arrest or poor cardiac condition. It is important to note that ICD patients with CAD feel more perplexed about their health condition. ICD device is a significant lifesaver and patients are required to live with their ICD for life (Josephson \& Wellens 2004). Further nursing education about ICD function and corresponding psychological reassurance can promote adaptation and acceptance of ICD.

Moreover, depression was found to have significant negative association with physical health in ICD patients with a history of CAD. It is important to note that depression increases physical disability but reduces physical tolerance (Wong et al. 2014). However, the mechanism of the interaction between depression and physical health remains unclear. Therefore, further investigation is recommended. Moreover, having occupation was found to be positively associated with physical health. It can be possibly due to the fact that patients who have occupation will have better sense of self-care independence and physical ability. It is important to note that ICD patients with a history of CAD would exhibit their ability of self-care and self-management. ICD device and CAD treatment improve patients' self-care ability and physical ability due to better control of symptoms and cardiac function. Moreover, having a history of hyperlipidemia was found to be associated with better physical health. It can be explained the fact that those patients will be instructed to control body weight and treated with lipid-lowering agents. It indicates that the specific participants with a history of hyperlipidemia in the present study were aware of their health problem and able to adhere to the treatment appropriately. As a result, their physical health will be enhanced. However, the mechanism by which occupation or hyperlipidemia is associated with HRQoL still remains unclear and further investigation is needed.

In the present study, a cross-sectional design was used but the design cannot provide causal inferential effects between factors and both psychological distress and HRQoL in physical and mental aspects among ICD patients with CAD. Although age was not found as a factor influencing physical and mental health among ICD patients with CAD in this study due to small sample size, it can be still a concern as the study patients were relatively old and old age in cardiac patients can heighten depression leading to decline HRQoL (Hamilton \& Carroll, 2004; Wong et al., 2014). As the study patients who were Chinese ICD patients with CAD were recruited, the results may not be generalizable. In addition, the effects of ICD shock on 
psychological distress and HRQoL are unclear due to small sample size with ICD shock $(n=2)$ although evidence showed that ICD shocks increase psychological distress and reduce physical and mental health (Carroll \& Hamilton 2005, Schron et al. 2012). Therefore, further investigation on this area is needed.

\section{CONCLUSION}

The present study has revealed that ICD patients with a history of CAD perceive relatively poorer physical health and heightened depression. Comparatively, this specific patient population may have more psychological concerns arising from their poor cardiac condition, possibility of cardiac attack episodes, and living with their ICD device for life. Factors associated with depression and anxiety as well as physical and mental health are identified in this study. Female gender was found to have positive association with anxiety; whereas, DM was found to have positive association with depression. Importantly, self-care dependence was the most significant factors associated with both anxiety and depression. Regarding factors associated with physical health and mental health, anxiety was found to be negatively associated with mental health; whereas, depression was found to have negative but having occupation and having a history of hyperlipidemia were found to have positive association with physical health. The study results are crucial to develop healthcare policy to early detect the risk group for early detection of poorer HRQoL and heightened psychological distress. Moreover, the results can also direct current cardiac care to develop appropriate intervention, such as psychological intervention, stress management, and cognitive behavioral intervention, for reducing anxiety and depression and optimizing physical and mental HRQoL in ICD patients with a history of CAD.

\section{ACKNOWLEDGMENT}

I would like to express my gratitude to the staff in the outpatient clinics at the study hospitals for their support and assistance during the period of data collection.

\section{REFERENCES}

[1] American Heart Association (AHA). Heart disease and stroke statistics 2017: At a glance. Retreived from http://www.heart.org/idc/groups/ahamahpublic/@wcm/@sop/@smd/documents/downloadable/ucm 491265.pdf.

[2] Bilge AK, Ozben B, Demircan S et al. Depression and anxiety status of patients with implantable cardioverter defibrillator and precipitating factors. PACE. 2016; 29, 6:619-626.

[3] Carroll DL, Hamilton GA. Long-term effects of implanted cardioverterdefibrillators on health status, quality of life and psychological state. Am J Crit Care. 2008; 17, 3: 222-231.

[4] Carroll DL, Hamilton GA Quality of life in implanted cardioverter defibrillator recipients: the impact of a device shock. Heart Lung. 2005; 34, 3:169-178.

[5] Centers for Disease Control and Prevention (CDC). Health-related quality of life concepts. 2016. Retrieved from http://www.cdc.gov/HRQOL/concept.htm.

[6] Center for Health Protection (CHP). Heart diseases. 2016. Retrieved from http://www.chp.gov.hk/en/content/9/25/57.html

[7] de Jonge P, Spijkerman TA, van den Brink RH, et al. Depression after myocardial infarction is a risk factor for declining health related quality of life and increased disability and cardiac complaints at 12 months. Heart. 2016; 92:32- 39 .
[8] Easton KL. Defining the concept of self-care. Rehabil Nurs. 1993; 18, 6: 384-387.

[9] Egede LE. Major depression in individuals with chronic medical disorders: prevalence, correlates and association with health resource utilization, lost productivity and functional disability. Gen Hosp Psychiat. 2007; 29: 409-416.

[10] Freedenberg V, Thomas SA, Friedmann E. Anxiety and depression in implanted cardioverter-defibrillator recipients and heart failure: a review. Heart Fail Clin. 2011; 7: 59-68.

[11] Friedmann E, Thomas SA, Inguito P, Kao CW, Metcalf M, Kelley FJ, Gottlieb SS. Quality of life and psychological status of patients with implantable cardioverter defibrillators. J Interv Card Electrophysiol. 2006; 17, 1: 65-72.

[12] Glassman AH. Depression and cardiovascular comorbidity. Dialogues in Clin Neurosci. 2007; 9, 1: 9-17.

[13] Goldenberg I., Moss A.J. Implantable device therapy. Prog Cardiovasc Dis. 2008; 50: 449-474.

[14] Hamilton, G. A. \& Carroll, D. L. (2004). The effects of age on quality of life in implantable cardioverter defibrillator recipients. Journal of Clinical Nursing, 13(2), 194-200.

[15] Issa SM, Hoeks SE, Scholte op Reimer, et al. Health-related quality of life predicts long-term survival in patients with peripheral artery disease. Vasc Med. 2010; 15,3:163-169.

[16] Josephson ME, Wellens HJ. Implantable defibrillators and sudden cardiac death. Circulation. 2004; 109, 22: 2685-269.

[17] Koplan BA. Ventricular Tachycardia and Sudden Cardiac Death. Mayo Clin Proc. 2009; 84, 3: 289-297.

[18] Kwok KM, Lee KLF, Lau CP, et al. Sudden cardiac death: prevention and treatment. HK Med J. 2003; 9: 357-362.

[19] Leung CM, Wing YK, Kwong PK, et al. Validation of the ChineseCantonese version of the Hospital Anxiety and Depression Scale and comparison with Hamilton Rating Scale of Depression. Acta Psychiatr Scand. 1999; 100: 456-461.

[20] Lichtman JH, Bigger T, Blumenthal JA, et al. Depression and coronary heart disease: Recommendations for screening, referral, and treatment. Circulation. 2008; 118: 1768-1775.

[21] Moryś JM, Bellwon J, Höfer S, et al. Quality of life in patients with coronary heart disease after myocardial infarction and with ischemic heart failure. Arch Med Sci. 2016 Apr 1, 12, 2:326-333.

[22] Porojan M, Poantả L, Dumitraşcu D.L. Assessing health related quality of life in diabetic patients. Romanian Journal of Internal Medicine. 2012; 50, 1: 27-31.

[23] Schron EB, Exner DV, Yao Q et al. Quality of life in the antiarrhythmics versus implantable defibrillators trial: impact of therapy and influence of adverse symptoms and defibrillator shocks. Circulation. 2002; 105, 5:589-594.

[24] Sears SF Jr, Conti JB. Quality of life and psychological functioning of ICD patients. Heart. 2002; 87, 5: 488-493.

[25] Doran DM. (2003) Nursing-sensitive Outcomes. State of the Science. Jones and Bartlett Learning, Boston, MA. Chapter Three Sidani S. Self care page 64-114.

[26] Smolderen KG. Coping after and acute myocardial infarction: The role of depression and anxiety. Am Coll Cardiol. 2017; Open Journal retrieved from http://www.acc.org/latest-incardiology/articles/2016/12/29/11/08/coping-after-an-acutemi?w nav=CI

[27] Thombs BD, Bass EB, Ford EB, et al. Prevalence of depression in survivors of acute myocardial infarction. J Gen Intern Med 2016; 21,1: 30-38.

[28] Thomas SA, Friedmann E, Kao CW et al. Quality of life and psychological status of patients with implantable cardioverter defibrillators. Am J Crit Care. 2016; 15, 4: 389-398.

[29] Tofighi S, Kiadaliri AA, Sadeghifar J, et al. Health-related quality of life among patients with coronary artery disease: A post-treatment follow-up study in Iran. Cardiol Res Pract. 2012; doi: 10.1155/2012/973974

[30] Tsu LV. (2012). Depression in cardiac patients: Underrecognized and undertreated. Pharmacist. 37 (11): HS-12-HS-15 Retrieved from 
https://www.uspharmacist.com/article/depression-in-cardiac-patientsunderrecognized-and-undertreated

[31] Unsar S, Sut N, Durna Z. (2007). Health-related quality of life in patients with coronary artery disease. J Cardiovasc Nurs; 22, 6: 501-507.

[32] Vazquez LD, Kuhl EA, Shea JB et al. Age-specific differences in women with implantable cardioverter defibrillators: an international multi center study. PACE. 2008; 31, 12: 1528-1534.

[33] Ware JE, Snow KK, Kosinski M., Gandek B. SF-36 Health Survey: Manual and Interpretation Guide. 1993. Boston: The Health Institute. New England Medical Center.

[34] Watkins LL, Koch GG, Sherwood A, et al. Association of anxiety and depression with all-cause mortality in individuals with coronary heart disease. J Am Heart Assoc. 2013; 2: 1-10.

[35] Welin C, Lappas G, Wilhelmsen L. Independent importance of psychosocial factors for prognosis after myocardial infarction. J Intern Med. 2000; 247, 6: 629-639.

[36] Wong FM, Sit JWH, Wong EM et al (2014) Factors associated with health-related quality of life among patients with implantable cardioverter defibrillator: identification of foci for nursing intervention. JAN. 70, 12: 2821-2834.

[37] World Health Organization (WHO) Cardiovascular diseases (CVDs). 2016

Retrieved

from http://www.who.int/mediacentre/factsheets/fs317/en/

[38] Yu DS, Thompson DR, Yu CM, et al. Assessing HRQL among Chinese patients with coronary heart disease: angina, myocardial infarction and heart failure. Int J Cardiol. 2009; 131: 384-94. 